\title{
Avaliação da Prevalência de Hipertensão Pulmonar na Esclerose Sistêmica $\left.{ }^{*}\right)$
}

\section{Prevalence of Pulmonary Hypertension in Systemic Sclerosis}

\author{
Ana Beatriz Cordeiro de Azevedo ${ }^{(1)}$, Percival D. Sampaio-Barros ${ }^{(2)}$, Rosália Moraes Torres ${ }^{(3)}$ e Caio Moreira ${ }^{(4)}$
}

\section{RESUMO}

Objetivo: Avaliar a prevalência da hipertensão pulmonar (HP) em pacientes com diagnóstico de esclerose sistêmica (ES) em acompanhamento num serviço universitário terciário. Métodos: Foram avaliados 57 pacientes com ES, em acompanhamento no Serviço de Reumatologia do HC-UFMG, através de exame clínico dirigido ao aparelho cardiorrespiratório, testes de função pulmonar e ecodopplercardiograma (ECO). Foram considerados critérios diagnósticos para HP: pressão sistólica de artéria pulmonar (PSAP) $\geq 40 \mathrm{mmHg}$ e/ou presença de outros sinais diretos ou indiretos de HP identificados durante o ECO. Resultados: Dezesseis pacientes (28\%) apresentaram diagnóstico de HP, sendo 13 com PSAP $\geq 40 \mathrm{mmHg}$ e $3 \mathrm{com}$ sinais indiretos de HP; 8 pacientes apresentaram HP isolada e $8 \mathrm{HP}$ secundária à fibrose pulmonar. Em 9 pacientes havia sinais sugestivos de cor pulmonale ao ECO. Destes pacientes, 6 apresentaram PSAP $\geq 40 \mathrm{mmHg}$ e 3 entre 35 e 40 mmHg; dentre eles, 1 era assintomático e 8 apresentavam sinais sugestivos de HP ao exame físico. Dentre as variáveis clínicas e laboratoriais observadas, somente encontramos correlação de HP com a velocidade de hemossedimentação (VHS) elevada $(\mathrm{p}=0,004)$. Conclusões: A prevalência de HP associada à ES encontrada neste estudo foi semelhante a outras da literatura, apesar das limitações nesta comparação. A investigação da HP através do ECO é uma prática acessível, de grande auxílio para o diagnóstico precoce do acometimento vascular pulmonar. No entanto, é necessário que seja revisto o ponto de corte da PSAP medida ao ECO para diagnóstico de HP relacionada à ES.

Palavras-chave: esclerose sistêmica, hipertensão pulmonar, ecocardiograma.

\begin{abstract}
Objective: To assess the prevalence of pulmonary arterial hypertension (PAH) in patients with diagnosis of systemic sclerosis (SSc) evaluated at a university tertiary service. Methods: Fifty-seven SSc patients attending the R heumatology outpatient clinic of HC-UFMG were studied by clinical assessment addressed to cardiopulmonary system, pulmonary function tests and Doppler echocardiography (ECO). The following criteria were considered for the diagnosis of PAH: pulmonary arterial systolic pressure $(P A S P) \geq 40 \mathrm{mmHg}$ and/or the presence of direct or indirect signs of PAH found during the ECO. Results: Sixteen patients (28\%) were found to have the diagnosis of $P A H$ at ECO, 13 with PASP $\geq 40 \mathrm{mmHg}$ and 3 with direct and indirect signs of $\mathrm{PAH} ; 8$ patients had isolated $\mathrm{PAH}$ and $8, \mathrm{PAH}$ secondary to pulmonary fibrosis. Nine patients had suggestive signs of cor pulmonale at ECO; 6 of these patients presented $P A S P \geq 40 \mathrm{mmHg}$ and 3 PASP between 35 and $40 \mathrm{mmHg}$; 1 of them was not symptomatic and 8 had clinical exam suggestive of PAH. Regarding the clinical and laboratory variables studied, it was observed statistical association between PAH and elevated erythrocyte sedimentation rate (ESR) $(p=0,004)$. Conclusions: There was a prevalence of PAH in SSc similar to that observed in the literature, in spite of limitations in this comparison. The assessment of PAH by ECO is accessible and practical; however, it is necessary to review the cut off of the PASP levels considered for the diagnosis of PAH in SSC, especially when considering an early diagnosis.
\end{abstract}

Keywords: systenic sclerosis, pulmonary arterial hypertension, doppler echocardiography.

\footnotetext{
* Trabalho realizado no Serviço de Reumatologia e no Setor de Ecocardiografia do Hospital das Clínicas da Universidade Federal de Minas Gerais (HC-UFMG). Projeto de Pesquisa integrante da 2. ${ }^{\text {a }}$ onda do Projeto Pronuclear da Sociedade Brasileira de Reumatologia. Este trabalho contou com o auxílio do Fundo de Amparo à Pesquisa da Sociedade Brasileira de Reumatologia. Recebido em 05/10/03. Aprovado, após revisão, em 24/01/04.

1. Médica Assistente do Serviço de Reumatologia do HC-UFMG.

2. Assistente-Doutor da Disciplina de Reumatologia da Faculdade de Ciências Médicas da Universidade Estadual de Campinas (FCM - UNICAMP). Orientador do Projeto Pronuclear da Sociedade Brasileira de Reumatologia.

3. Professora Assistente do Departamento de Clínica Médica da Faculdade de Medicina da UFMG. Coordenadora do Setor de Ecocardiografia do Serviço de Cardiologia e Cirurgia Vascular do HC-UFMG.

4. Médico Assistente do Serviço de Reumatologia do HC-UFMG.
}

Endereşo para correspondência: Dra. Ana Beatriz C. Azevedo. Rua Cardoso de Almeida, 457, ap. 144, CEP 05013-000, Perdizes, São Paulo, SP. E-mail: anabiazevedo@hotmail.com 


\section{INTRODUÇÃO}

A esclerose sistêmica (ES) é uma doença inflamatória do tecido conjuntivo, associada a alterações vasculares, caracterizada clinicamente pelo espessamento cutâneo, fenômeno de Raynaud e diferentes formas de manifestações viscerais, que acometem principalmente o trato gastrintestinal, os pulmões, o coração e os rins ${ }^{(1)}$. $\mathrm{O}$ acometimento pulmonar ocorre em mais de $70 \%$ dos pacientes com ES, sendo a segunda manifestação visceral mais freqüente, suplantado somente pelo comprometimento esofágico. As principais formas de acometimento pulmonar na ES são a doença intersticial pulmonar, também conhecida por alveolite fibrosante ou fibrose pulmonar, e a doença vascular pulmonar que leva à hipertensão pulmonar isolada ${ }^{(2,3)}$.

A hipertensão pulmonar (HP) é uma causa importante de morbimortalidade na ES. A freqüência da HP na ES é incerta, variando entre $5 \%$ e $50 \%$, dependendo da metodologia empregada na sua investigação ${ }^{(4)}$. Geralmente, os sinais e sintomas relacionados à HP na ES são insidiosos, principalmente nas fases iniciais desta complicação, e até um terço dos casos podem ser assintomáticos ${ }^{(1-3)}$. O ecodopplercardiograma bidimensional transtorácico é um exame útil para a medida não invasiva da pressão em artéria pulmonar, com sensibilidade de $90 \%$ e especificidade de $75 \%$ para o diagnóstico de HP na ES, através de medidas indiretas pelo jato de regurgitação tricúspide, considerando valores anormais para pressão sistólica de artéria pulmonar $(\mathrm{PSAP}) \geq 30 \mathrm{mmHg}^{(5)}$.

O presente estudo tem por objetivo avaliar a prevalência de HP em pacientes com diagnóstico de ES em acompanhamento num serviço universitário terciário, utilizando como método de diagnóstico o ecodopplercardiograma bidimensional transtorácico.

\section{MATERIAIS E MÉTODOS}

Pacientes: Foi realizada uma avaliação transversal dos pacientes com diagnóstico de ES em acompanhamento no Serviço de Reumatologia do Hospital das Clínicas da Universidade Federal de Minas Gerais (HC-UFMG), no período de novembro de 2001 a março de 2003. Todos os pacientes preenchiam os critérios para ES do Colégio Americano de Reumatologia ${ }^{(6)}$, e foram subdivididos em ES limitada e ES difusa de acordo com os critérios de LeRoy e colaboradores ${ }^{(7)}$. Os pacientes foram submetidos a questionário e exame físico dirigidos para a avaliação de sinais e sintomas cardiorrespiratórios e encaminhados para a realização de ecodopplercardiograma bidimensional trans- torácico (ECO) e provas de função pulmonar após assinarem termo de consentimento para o estudo.

Ecodopplercadiograma: Os exames de ecodopplercardiograma bidimensional transtorácico foram realizados por um único profissional experiente, em aparelho de ecocardiograma Sonos 1500 HP, com transdutor 2,5/2,0. Durante o ECO, foram observadas as características das câmaras cardíacas, alterações de contratilidade e relaxamento diastólico, função sistólica do ventrículo esquerdo (VE), alterações valvares e do pericárdio, além da medida da PSAP na presença de regurgitação tricúspide. Diante da impossibilidade de realização desta medida, foram pesquisados outros sinais de HP através da medida da pressão diastólica em artéria pulmonar na presença de insuficiência pulmonar, medida do tempo de aceleração (TAC) do fluxo pulmonar, investigação de presença de incisura mesodiastólica no registro doppler da valva pulmonar, medida do tempo de velocidade máxima/tempo de ejeção, cálculo do intervalo entre o fechamento da artéria pulmonar e abertura da tricúspide (tempo de relaxamento isovolumétrico do VD), medida do quociente entre o tempo de pré-ejeção/tempo de ejeção no fluxo pulmonar e cálculo da pressão média em artéria pulmonar.

A presença de hipertensão pulmonar foi definida pela medida de PSAP estimada pelo ecodopplercardiograma $\geq 40 \mathrm{mmHg}$, através de medidas realizadas pelo jato de regurgitação tricúspide, utilizando a equação de Bernouli $\left(\mathrm{PSAP}=4 \mathrm{VTR}^{2}+\mathrm{PAD}\right)$, com pressão estimada de átrio direito (PAD) igual a $14 \mathrm{mmHg}$ e/ou presença de outros sinais de HP diretos ou indiretos. Foram considerados sinais diretos de $\mathrm{HP}$, que não $\mathrm{PSAP} \geq 40 \mathrm{mmHg}$ : pressão diastólica em artéria pulmonar $\geq 25 \mathrm{mmHg}$, TAC $<100 \mathrm{~ms}$, presença de incisura mesodiastólica no registro doppler da valva pulmonar, tempo de velocidade máxima/tempo de ejeção $<0,36$, prolongamento do tempo de relaxamento isovolumétrico do ventrículo direito, quociente entre o tempo de pré-ejeção/tempo de ejeção no fluxo pulmonar $\geq 0,34$ e pressão média de artéria pulmonar $\geq 30 \mathrm{mmHg}$. Foram considerados sinais indiretos de HP: a presença de dilatação de ventrículo direito (VD) associado à regurgitação tricúspide e/ou pulmonar, dilatação do átrio direito, dilatação do tronco da artéria pulmonar, valva pulmonar com rampa "a" retificada, espessamento do lado direito do septo interventricular, protusão do septo interventricular para dentro do VE durante a diástole e hipertrofia da parede livre do $\mathrm{VD}^{(8)}$. Os pacientes que apresentassem outras alterações cardiovasculares passíveis de desencadear HP foram excluídos da análise estatística. 
Provas de função pulmonar: Foram realizadas espirometria simples com plestimografia e medida da difusão de monóxido de carbono em aparelho Collins DS2plus no laboratório de função pulmonar do HC-UFMG, que segue as orientações do Consenso Brasileiro de Espirometria ${ }^{(9)}$.

Tomografia computadorizada de alta resolução de tórax (TCAR): Os pacientes com diagnóstico de HP que não tinham doença intersticial pulmonar conhecida foram encaminhados para realização de TCAR para diagnóstico e/ou exclusão de fibrose pulmonar associada à HP. Os exames de TCAR foram realizados em tomógrafo SOMATON ART - Siemens, com cortes de $2 \mathrm{~mm}$, a intervalos de $10 \mathrm{~mm}$, com janela de $1200 \mathrm{UH}$ e centro de $-700 \mathrm{UH}$. Diante da presença de alterações intersticiais foram realizados também cortes em decúbito ventral.

Análise estatística: Foram realizados descrição de médias e medianas e testes $t$ de Student, $X^{2}$ de Pearson e ANOVA para a pesquisa de possíveis fatores associados à presença de HP.

\section{RESULTADOS}

Foram analisados inicialmente 60 pacientes com diagnóstico de ES, sendo excluídos dois pacientes com valvulopatia mitral reumática e um com prolapso mitral. Dentre os 57 pacientes avaliados, 32 tinham ES difusa, 24 ES limitada e 1 ES sine scleroderma. A média de idade foi de 42,6 anos (variando de 18 a 70 anos, com desvio-padrão (DP) \pm 13 ,1), enquanto que a duração média da doença foi de 9,6 anos (variando de um a 30 anos, com DP $\pm 6,9)$. O perfil clínico e epidemiológico dos pacientes está descrito na Tabela 1.

A prevalência de HP no presente estudo foi de 28\% (16/ 57 casos), sendo oito casos de HP isolada (quatro com ES difusa e quatro ES limitada) e oito secundários à fibrose pulmonar (cinco com ES difusa, dois com ES limitada e um com ES sine scleroderma) (Tabela 2). A medida da PSAP não foi possível em 4 pacientes, por causa da ausência de regurgitação tricúspide; 1 destes pacientes (ES difusa) apresentava alteração no TAC compatível com HP, sendo a PSAP estimada através desta medida igual a $48,5 \mathrm{mmHg}$; os outros 3 pacientes sem jato de regurgitação tricúspide apresentaram outros sinais diretos e/ou indiretos de HP. As características dos 16 pacientes esclerodérmicos com HP estão especificadas na Tabela 3.

Nove pacientes com HP apresentaram sinais sugestivos de cor pulmonale ao ECO; destes, 3 apresentavam PSAP entre 35 e $40 \mathrm{mmHg}$ e 6 PSAP $\geq 40 \mathrm{mmHg}$. Um dos 16 pacientes com HP era assintomático, apesar da PSAP igual a $40 \mathrm{mmHg}$ associada à presença de dilatação de átrio e
TABELA 1

Perfil clínico e ePidemiológico

\begin{tabular}{|c|c|}
\hline $\begin{array}{l}\text { Idade } \\
\text { Média } \\
\text { Mediana } \\
\text { desvio padrão }\end{array}$ & $\begin{array}{c}\text { 42,6 anos } \\
43 \text { anos } \\
+/-13,1 \text { anos }\end{array}$ \\
\hline \multicolumn{2}{|l|}{ Sexo } \\
\hline Masculino & $8(14 \%)$ \\
\hline Feminino & $49(86 \%)$ \\
\hline \multicolumn{2}{|l|}{ Cor } \\
\hline Brancos & $14(25 \%)$ \\
\hline Pardos & $28(49 \%)$ \\
\hline Negros & $15(26 \%)$ \\
\hline \multicolumn{2}{|l|}{ Forma cutânea } \\
\hline Difusa & $32(56 \%)$ \\
\hline Limitada & $24(42 \%)$ \\
\hline sem pele & $1(2 \%)$ \\
\hline \multicolumn{2}{|l|}{ Tempo de doença } \\
\hline Média & 9,6 anos \\
\hline Mediana & 8 anos \\
\hline desvio padrão & $+/-6,9$ anos \\
\hline \multicolumn{2}{|l|}{ Tempo de diagnóstico } \\
\hline Média & 6,9 anos \\
\hline Mediana & 6 anos \\
\hline desvio padrão & $+/-6,5$ anos \\
\hline \multicolumn{2}{|l|}{ Tabagismo } \\
\hline Sim & $5(9 \%)$ \\
\hline Não & $38(67 \%)$ \\
\hline ex-tabagista & $14(24 \%)$ \\
\hline \multicolumn{2}{|l|}{ HAS } \\
\hline Sim & $18(32 \%)$ \\
\hline Não & $39(68 \%)$ \\
\hline Fenômeno de Raynaud & $56(98 \%)$ \\
\hline Telangiectasias & $48(84 \%)$ \\
\hline Úlceras de polpas digitais & $46(81 \%)$ \\
\hline \multicolumn{2}{|l|}{ Fibrose pulmonar } \\
\hline Presente & $30(53 \%)$ \\
\hline Ausente & $25(44 \%)$ \\
\hline Desconhecida & $2(3 \%)$ \\
\hline \multicolumn{2}{|l|}{ FAN } \\
\hline Positivo & $50(88 \%)$ \\
\hline Negativo & $7(12 \%)$ \\
\hline \multicolumn{2}{|l|}{ Anti-centrômero } \\
\hline Positivo & $8(14 \%)$ \\
\hline Negativo & $47(82 \%)$ \\
\hline Desconhecido & $2(4 \%)$ \\
\hline \multicolumn{2}{|l|}{ Anti-Scl70 } \\
\hline Positivo & $3(5 \%)$ \\
\hline Negativo & $50(88 \%)$ \\
\hline Desconhecido & $4(7 \%)$ \\
\hline \multicolumn{2}{|l|}{ Anti-RNP } \\
\hline Positivo & $22(39 \%)$ \\
\hline Negativo & $30(53 \%)$ \\
\hline Desconhecido & $5(8 \%)$ \\
\hline \multicolumn{2}{|l|}{ VHS } \\
\hline Média & 33,9 \\
\hline Mediana & 27 \\
\hline desvio padrão & $+/-25,2$ \\
\hline \multicolumn{2}{|l|}{ DLCO } \\
\hline Média & $75,5 \%$ \\
\hline Mediana & $75 \%$ \\
\hline desvio padrão & $+/-22,2$ \\
\hline Total & 57 pacientes \\
\hline
\end{tabular}


ventrículo direitos e redução da DLCO (64\%). Oito pacientes com HP apresentavam hiperfonese da $2^{\mathrm{a}}$ bulha, todos com PSAP $\geq 40 \mathrm{mmHg}$; destes, 1 apresentava também sopro de insuficiência tricúspide e impulsão do VD à palpação do precórdio e 1 outro paciente edema de membros inferiores. Dos 16 pacientes com HP, em 2 não foi possível a medida da DLCO em razão da dificuldade em realizar as manobras necessárias para o exame (um por não compreender adequadamente as instruções e outro por distúrbio restritivo grave).
TABELA 2

PrevalÊNCIA DE HP

\begin{tabular}{lcccc}
\hline & \multicolumn{3}{c}{ Forma Clínica } & Total \\
\hline HP & $\begin{array}{c}\text { Difusa } \\
(\mathrm{n}=32)\end{array}$ & $\begin{array}{c}\text { Limitada } \\
(\mathrm{n}=24)\end{array}$ & $\begin{array}{c}\text { Sem pele } \\
(\mathrm{n}=1)\end{array}$ & $\mathrm{N}=57$ \\
\hline Isolada & 4 & 4 & 0 & $8(14 \%)$ \\
Sencundária à & 5 & 2 & 1 & $8(14 \%)$ \\
Fibrose Pulmonar & & & & $16(28 \%)$ \\
\hline Total & 9 & 6 & 1 &
\end{tabular}

TABELA 3

Descriç̃̃o dos CASOS DE HP

\begin{tabular}{|c|c|c|c|c|c|c|c|c|c|}
\hline Caso & Sexo & $\begin{array}{l}\text { Idade } \\
\text { (anos) }\end{array}$ & $\begin{array}{c}\text { Tempo de doença } \\
\text { (anos) }\end{array}$ & Forma cutânea & Dispnéia & $\begin{array}{l}\text { Alterações ao } \\
\text { exame físico } \\
\text { sugestiva de HP }\end{array}$ & $\begin{array}{c}\text { PSAP } \\
(\mathrm{mmHg})\end{array}$ & $\begin{array}{c}\text { Alterações de } \\
\text { câmaras direitas } \\
\text { ao ECO }\end{array}$ & $\begin{array}{l}\text { Fibrose } \\
\text { pulmonar }\end{array}$ \\
\hline 1 & Fem & 25 & 14 & difusa & $\operatorname{sim}$ & $\operatorname{sim}$ & 48,5 & não & não \\
\hline 2 & Fem & 57 & 7 & limitada & $\operatorname{sim}$ & $\operatorname{sim}$ & 40 & não & não \\
\hline 3 & Fem & 47 & 3 & difusa & sim & não & 48 & não & não \\
\hline 4 & Fem & 30 & 5 & limitada & $\operatorname{sim}$ & $\operatorname{sim}$ & 56 & sim & não \\
\hline 5 & Masc & 23 & 4 & difusa & não & não & 40 & $\operatorname{sim}$ & não \\
\hline 6 & Fem & 43 & 2 & difusa & sim & $\operatorname{sim}$ & 44 & não & não \\
\hline 7 & Fem & 49 & 18 & limitada & sim & não & 39 & sim & não \\
\hline 8 & Masc & 29 & 2 & limitada & sim & $\operatorname{sim}$ & 41 & não & não \\
\hline 9 & Fem & 40 & 5 & difusa & sim & não & 42 & não & sim \\
\hline 10 & Fem & 42 & 9 & difusa & $\operatorname{sim}$ & não & 47 & não & $\operatorname{sim}$ \\
\hline 11 & Fem & 50 & 10 & sem pele & $\operatorname{sim}$ & não & 50 & sim & $\operatorname{sim}$ \\
\hline 12 & Masc & 63 & 7 & limitada & sim & não & 39 & sim & sim \\
\hline 13 & Fem & 68 & 3 & difusa & sim & sim & 58 & sim & $\operatorname{sim}$ \\
\hline 14 & Fem & 46 & 10 & limitada & sim & $\operatorname{sim}$ & 40,6 & sim & $\operatorname{sim}$ \\
\hline 15 & Fem & 56 & 16 & difusa & sim & $\operatorname{sim}$ & 58 & sim & $\operatorname{sim}$ \\
\hline 16 & Fem & 37 & 8 & difusa & $\operatorname{sim}$ & não & 37 & $\operatorname{sim}$ & $\operatorname{sim}$ \\
\hline
\end{tabular}

Analisando-se os valores da PSAP, 17 pacientes tinham PSAP $\geq 35$ e $<40 \mathrm{mmHg}$ (Tabela 4 ). Três deles ( 2 com ES limitada e 1 com ES difusa) apresentavam queixa clínica de dispnéia e alterações em câmaras direitas ao ECO sugestivas de cor pulmonale, sendo classificados como portadores de HP; 2 destes pacientes tinham alterações tomográficas e/ou espirométricas compatíveis com doença intersticial pulmonar, sem redução desproporcional da DLCO, enquanto que o outro paciente (ES limitada) não apresentava evidências de doença intersticial pulmonar e tinha DLCO normal (78\%). Dentro deste grupo (PSAP $\geq 35$ e $<40 \mathrm{mmHg}$ ), 4 pacientes não conseguiram realizar manobras adequadas para a medida de DLCO; entre os que a realizaram, apenas 1 paciente (assintomático, com ES difusa) apresentava redução isolada da DLCO (60\%), sem alterações à TCAR do tórax. Vinte e um pacientes apresentavam PSAP $\geq 30$ e $<35 \mathrm{mmHg}$, enquanto somente 2 pacientes (1 com ES difusa e outro com ES limitada) apresentavam PSAP $<30 \mathrm{mmHg}$.

Analisando-se os fatores epidemiológicos, clínicos e laboratoriais, a ocorrência da HP correlacionou-se somente com a velocidade de hemossedimentação (VHS) elevada $(p=0,004)$ (Tabela 5). Não foi encontrada correlação entre HP e redução da difusão de $\operatorname{CO}(p=0,811)$. 
TABELA 4

Valores de PSAP E alterações de cÂmaras direitas

\begin{tabular}{lcc}
\hline PSAP & Número de Pacientes & Alterações de Câmaras Direitas ao EC0 \\
& $\mathrm{N}=57$ & $\mathrm{~N}=9 / 57(15,8 \%)$ \\
\hline Não mensurado pelo jato de regurgitação tricúspide & $4^{*}(7 \%)$ & $0(0 \%)$ \\
$<30 \mathrm{mmHg}$ & $2(3 \%)$ & $0(0 \%)$ \\
$\geq 30 \mathrm{mmHg} \mathrm{e}<35 \mathrm{mmHg}$ & $21(37 \%)$ & $0(0 \%)$ \\
$\geq 35 \mathrm{mmHg} \mathrm{e}<40 \mathrm{mmHg}$ & $17(30 \%)$ & $3(5,3 \%)$ \\
$\geq 40 \mathrm{mmHg}$ & $13(23 \%)$ & $6(10,5 \%)$ \\
Total & $57(100 \%)$ & $9(15,8 \%)$ \\
\hline
\end{tabular}

0bs: * : destes 3 não apresentavam alterações diretas e ou indiretas sugestivas de HP e 1 apresentava TAC < 100ms, sugestivo de HP.

TABELa 5

Características CLÍNICAS E LABORATORIAIS DOS PACIENTES COM E SEM HP

\begin{tabular}{|c|c|c|}
\hline & Com HP $(n=16)$ & Sem HP $(n=41)$ \\
\hline $\begin{array}{l}\text { Idade } \\
\qquad \text { Média } \\
\text { Desvio padrão }\end{array}$ & $\begin{array}{c}\text { 44,1 anos } \\
+/-13,2 \text { anos }\end{array}$ & $\begin{array}{c}\text { 42,1 anos } \\
+/-13,2 \text { anos }\end{array}$ \\
\hline Mulheres (\%) & $13(81 \%)$ & $36(88 \%)$ \\
\hline $\begin{array}{l}\text { Tempo de doença } \\
\text { Média } \\
\text { Desvio padrão }\end{array}$ & $\begin{array}{c}7,7 \text { anos } \\
+/-4,9 \text { anos }\end{array}$ & $\begin{array}{c}10,4 \text { anos } \\
+/-7,4 \text { anos }\end{array}$ \\
\hline $\begin{array}{l}\text { Formas clínicas } \\
\text { Difusa } \\
\text { Limitada } \\
\text { Sine scleroderma }\end{array}$ & $\begin{array}{c}9(56 \%) \\
6(38 \%) \\
1(6 \%)\end{array}$ & $\begin{array}{c}23(56 \%) \\
18(44 \%) \\
0(0 \%)\end{array}$ \\
\hline HAS & $6(37 \%)$ & $12(29 \%)$ \\
\hline Tabagistas & $1(6 \%)$ & $4(10 \%)$ \\
\hline Fenômeno de Raynaud & $16(100 \%)$ & $40(98 \%)$ \\
\hline Úlceras de polpas digitais & $13(81 \%)$ & $33(81 \%)$ \\
\hline Telangiectasias & $14(87 \%)$ & $34(83 \%)$ \\
\hline Fibrose Pulmonar & $8(50 \%)$ & $22(54 \%)$ \\
\hline FAN & $14(87 \%)$ & $35(85 \%)$ \\
\hline Anti-centrômero & $2(15 \%)$ & $6(15 \%)$ \\
\hline Anti-Scl 70 & $1(8 \%)$ & $2(6 \%)$ \\
\hline Anti-RNP & $7(54 \%)$ & $14(41 \%)$ \\
\hline $\begin{array}{l}\text { VHS }^{\dagger} \\
\qquad \text { Média } \\
\text { Desvio padrão }\end{array}$ & $\begin{aligned} & 46 \\
+ & /-28\end{aligned}$ & $\begin{array}{c}29 \\
+/-23\end{array}$ \\
\hline $\begin{array}{l}\text { DLCO } \\
\qquad \begin{array}{l}\text { Média } \\
\text { Desvio padrão }\end{array}\end{array}$ & $\begin{array}{c}76,2 \% \\
+/-26,2 \%\end{array}$ & $\begin{array}{c}75,2 \% \\
+/-20,7 \%\end{array}$ \\
\hline
\end{tabular}

Obs: ${ }^{\mathrm{t} P}=0,004$.

Rev Bras Reumatol, v. 44, n. 1, p. 31-9, jan./fev., 2004

\section{DISCUSSÃO}

Desde o advento dos inibidores da enzima conversora da angiotensina para o tratamento da crise renal esclerodérmica, as manifestações pulmonares passaram a ocupar o primeiro lugar entre as causas de mortalidade nos pacientes com $\mathrm{ES}^{(1-3)}$. Uma análise das causas de óbito de 2.000 pacientes com ES acompanhados na Universidade de Pittsburgh nos últimos 20 anos revelou que 211 pacientes haviam falecido de causas pulmonares, sendo 113 por hipertensão pulmonar isolada e 98 por fibrose pulmonar; estes óbitos correspondem a 21,5\% das 981 mortes por ES e $44 \%$ das mortes relacionadas à doença naquele serviço ${ }^{(10)}$. Como a manifestação clínica do acometimento pulmonar na ES costuma ser tardia, esforços têm sido direcionados para a identificação de variáveis clínicas e laboratoriais que permitam a identificação de pacientes com maior risco para o desenvolvimento de doença intersticial e vascular pulmonar, bem como para o diagnóstico precoce destas complicações.

A etiopatogenia da HP na ES é bastante complexa e parece compreender uma seqüência de eventos a partir de lesão associada à disfunção do endotélio pulmonar, com conseqüente vasoespasmo, remodelação vascular e predisposição à trombose em pequenos vasos pulmonares. Atualmente, acredita-se que a lesão endotelial nas pequenas artérias e arteríolas pulmonares seja a alteração inicial no processo que levará ao vasoespasmo e à remodelação vascular ${ }^{(4,11)}$. Um aumento nos níveis circulantes de endotelina-1, um peptídeo vasoconstritor, tem sido correlacionado com as manifestações vasculares e fibróticas da ES, especialmente em pacientes com ES limitada e HP ou crise renal esclerodérmica, colaborando com a teoria da lesão/ disfunção endotelial ${ }^{(12)}$. 
Na ES limitada, a HP geralmente ocorre como manifestação isolada, sendo subclínica até que o dano arterial resulte em dispnéia, nas fases intermediária e tardia da doença ${ }^{(1-3)}$. $\mathrm{Na}$ ES difusa, a HP isolada é rara, estando normalmente associada à fibrose pulmonar avançada, levando ao desenvolvimento de sintomas cardiopulmonares adicionais. Alguns auto-anticorpos foram correlacionados à presença da HP nas diferentes formas clínicas da ES, como o anti$\mathrm{Th} / \mathrm{To}^{(13)}$ com a ES limitada e o anti-U3RNP com a forma difusa $^{(14)}$. No entanto, a pesquisa destes auto-anticorpos ainda não está disponível na rotina dos serviços universitários em nosso país.

A redução da difusão de $\mathrm{CO}$ desproporcional à queda da capacidade vital forçada (CVF) tem sido considerada como importante fator preditivo de doença vascular pulmonar na ES. Através de estudo retrospectivo caso-controle, Steen observou que pacientes com ES e HP apresentavam valores de DLCO acentuadamente diminuídos quando comparados aos controles num período de cerca de 4,5 anos antes do diagnóstico de HP $(\mathrm{P}<0,0001)^{(15)}$. Uma relação CVF/DLCO entre 1,6 e 1,8 pode ajudar a identificar os pacientes com maior probabilidade de doença vascular pulmonar ${ }^{(10)}$. O fato de não encontrarmos associação entre HP e níveis baixos de DLCO reflete as limitações do desenho deste estudo e do ECO como método isolado de diagnóstico de HP na ES. Na população estudada, observamos casos de fibrose pulmonar grave, sem HP secundária, associados à redução significativa da DLCO, mas proporcional à queda da CVF, bem como pacientes com redução isolada da DLCO sem critérios para o diagnóstico de HP ou doença intersticial pulmonar. Um acompanhamento prospectivo destes pacientes seria mais adequado para avaliar melhor esta associação.

A associação de HP com VHS elevada observada neste estudo também foi encontrada por Yamane et al., que descreveram uma prevalência de HP igual a 16\% (6 HP secundária à fibrose pulmonar e 14 HP isolada) numa população de 125 pacientes, utilizando valores de PSAP $\geq 40 \mathrm{mmHg}$ ao ECO para diagnóstico de HP, como neste estudo $^{(16)}$. O aumento na VHS também tem sido associado à maior gravidade e mortalidade relacionadas à doença intersticial pulmonar na $\mathrm{ES}^{(17)}$. A associação de HP com o aumento da VHS, apesar de não ter sido confirmada em outros estudos, é apontado por alguns autores como sugestivo da presença de atividade inflamatória pulmonar e como um possível indicador de pior evolução, tanto do acometimento intersticial quanto vascular pulmonar ${ }^{(18)}$. Porém, até o momento, o diagnóstico precoce da HP relacionada à ES ainda depende de uma rotina de investigação que inclui a combinação de testes de função pulmonar e ecodopplercardiograma.

A primeira série de casos de pacientes com ES e HP foi descrita por Sackner em 1964 $4^{(19)}$; a partir daí outras séries foram descritas na literatura ${ }^{(14,16,20-26)}$ (Tabela 6). A comparação dos achados do presente estudo com outros da literatura se torna difícil em razão das diferenças entre as populações estudadas, a metodologia empregada para a investigação da HP e os critérios utilizados para definir a sua presença. Os estudos que utilizaram o cateterismo (CAT) de ventrículo direito para diagnóstico demonstraram uma prevalência de $5 \%$ a $12 \%$, enquanto aqueles que empregaram o ECO revelaram taxas de prevalência mais alta, entre $13 \%$ e $35 \%{ }^{(14,16,19-25)}$.

Considera-se que o CAT do ventrículo direito seja o método diagnóstico mais fidedigno para avaliar a prevalência da HP relacionada à ES. Contudo, os dados provenientes destes estudos podem subestimar a prevalência desta complicação, uma vez que os pacientes encaminhados para a realização deste exame via de regra são sintomáticos $^{(10)}$. Quanto aos estudos com ECO, bastante explorado pelas suas vantagens em relação ao CAT de ventrículo direito, a maior dificuldade para a comparação dos resultados é o ponto de corte do nível da PSAP considerado como sugestivo de HP. O valor considerado como anormal na maioria destes estudos é diferente do valor recomendado no último consenso sobre HP, realizado em 1998. De acordo com este Consenso, somente valores de PSAP $\geq 40 \mathrm{mmHg}$ deveriam ser considerados para diagnóstico de HP pelo $\mathrm{ECO}^{(27)}$. Com a preocupação de diagnosticar precocemente o dano vascular pulmonar na ES, em vista da sua gravidade, o valor de PSAP $\geq 30 \mathrm{mmHg}$ ao ECO tem sido utilizado por alguns autores, destacando-se o estudo de Denton et al. ${ }^{(5)}$. No entanto, a crítica que se faz quanto à utilização deste ponto de corte é que este poderia implicar numa superestimativa dos casos de HP relacionados à ES.

No Brasil, estudo de Sampaio-Barros et al. ${ }^{(21)}$, analisando prospectivamente 95 pacientes esclerodérmicos submetidos ao ECO, encontrou uma prevalência de 30\% de HP, considerando PSAP $\geq 30 \mathrm{mmHg} ; 19$ casos foram associados à fibrose pulmonar e 10 considerados como manifestação pulmonar isolada (todos com ES limitada).

MacGregor et al. relataram a prevalência cumulativa de HP igual a 13\%, considerando a presença de HP com valores de PSAP $\geq 30 \mathrm{mmHg}$, medida pelo jato de regurgitação tricúspide ao ECO. Porém, a análise retrospectiva da progressão da HP durante cinco anos neste estudo revelou que a maioria dos pacientes permanecia com valores de PSAP 
TABela 6

PRINCIPAIS DADOS SOBRE A PREVALÊNCIA DA HP NA ES

\begin{tabular}{|c|c|c|c|c|}
\hline Autores/Ano de publicação & Tipo de estudo & $\begin{array}{l}\text { Características da } \\
\text { população estudada }\end{array}$ & $\begin{array}{c}\text { Critérios de diagnóstico } \\
\text { para HP }\end{array}$ & Prevalência (\%) \\
\hline Stupi et al 1986 & Retrospectivo & $\begin{array}{c}673 \\
\text { (CREST) }\end{array}$ & $\begin{array}{c}\mathrm{mPAP}>20 \mathrm{mmHg} \\
\text { PSAP/PDAP }>30 / 15 \mathrm{mmHg} \text { e } \\
\text { PCP }<12 \mathrm{mmHg} \text { no CAT e/ou } \\
\text { quadro clínico de cor pulmonale }\end{array}$ & $\begin{array}{c}9 \% \text { (HP isolada e } 2^{\mathrm{a}} \text { a } \\
\text { fibrose pulmonar) }\end{array}$ \\
\hline Sampaio-Barros et al 1995 & Prospectivo & $\begin{array}{c}95 \\
\text { (forma difusa e limitada) }\end{array}$ & $\mathrm{PSAP} \geq 30 \mathrm{mmHg}$ ao ECO & $\begin{array}{c}30 \% \text { (10 HP isolada e } 19 \\
2^{\mathrm{a}} \text { a fibrose pulmonar) }\end{array}$ \\
\hline Koh et al 1996 & Retrospectivo & $\begin{array}{c}344 \\
\text { (140 forma difusa e } \\
204 \text { limitada) }\end{array}$ & $\begin{array}{c}\mathrm{mPAP} \geq 25 \mathrm{mmHg} \text { com } \\
\text { PCP } \leq 12 \mathrm{~mm} \mathrm{mg} \text { no CAT ou } \\
\text { PSAP } \geq 35 \mathrm{mmHg} \text { no ECO ou } \\
\text { evidências de dilatação de } \\
\text { ventrículo direito e artéria } \\
\text { pulmonar e/ou regurgitação } \\
\text { tricúspide ou movimento } \\
\text { paradoxal do septo } \\
\text { interventricular ao ECO }\end{array}$ & $\begin{array}{l}4,9 \% \text { (HP isolada e } 2^{\mathrm{a}} \text { a } \\
\text { fibrose pulmonar) }\end{array}$ \\
\hline Battle et al 1996 & Transversal & $\begin{array}{c}34 \\
\text { (29 com a forma difusa } \\
\text { e } 5 \text { limitada) }\end{array}$ & $\mathrm{PSAP} \geq 30 \mathrm{mmHg}$ ao ECO & $\begin{array}{l}35 \% \text { (HP isolada e } 2^{\mathrm{a}} \text { a } \\
\text { fibrose pulmonar) }\end{array}$ \\
\hline Murata et al 1997 & Prospectivo & $\begin{array}{c}135 \\
\text { (forma limitada e difusa) } \\
\text { com ou sem sobreposição } \\
\text { com outra doença do } \\
\text { tecido conjuntivo }\end{array}$ & $\begin{array}{c}\text { PSAP } \geq 40 \mathrm{mmHg} \text { e/ou sinais } \\
\text { de sobrecarga de VD ao ECO ou } \\
\text { mPAP } \geq 20 \mathrm{mmHg} \text { ao CAT }\end{array}$ & $\begin{array}{l}22 \% \text { (HP isolada e } 2^{\mathrm{a}} \text { a } \\
\text { fibrose pulmonar) }\end{array}$ \\
\hline Yamane et al 2000 & Transversal & $\begin{array}{l}125 \\
\text { (55 forma difusa e } \\
70 \text { limitada) }\end{array}$ & $\mathrm{PSAP} \geq 40 \mathrm{mmHg}$ ao $\mathrm{ECO}$ & $\begin{array}{c}16 \% \text { (HP isolada e } 2^{\mathrm{a}} \text { a } \\
\text { fibrose pulmonar) }\end{array}$ \\
\hline Azevedo et al 2004 & Transversal & $\begin{array}{c}57 \\
\text { (32 forma difusa, } 24 \text { limitada } \\
\text { e } 1 \text { sine scleroderma) }\end{array}$ & $\begin{array}{l}\text { PSAP } \geq 40 \mathrm{mmHg} \text { ou } \\
\text { evidências diretas e/ou } \\
\text { indiretas de HP ao ECO }\end{array}$ & $\begin{array}{c}28 \% \text { (8 HP isolada e } 82^{\mathrm{a}} \\
\text { fibrose pulmonar) }\end{array}$ \\
\hline
\end{tabular}

estáveis, enquanto quase um terço apresentou diminuição dos níveis de PSAP. Apesar disto, a presença de PSAP $\geq 30$ $\mathrm{mmHg}$ em qualquer momento do acompanhamento foi associada a um risco de morte de $20 \%$ em 20 meses, sendo maior naqueles casos com valores de PSAP mais elevados à apresentação da HP, nos que evoluíram com elevação da PSAP ao longo do seguimento, em pacientes do sexo masculino, mais velhos e com ES limitada ${ }^{(24)}$.
Mukerjee et al. encontraram HP em 89 (12\%) dentre 722 pacientes acompanhados durante quatro anos, com sobrevida média de $81 \%, 63 \%$ e $56 \%$ em um, dois e três anos, respectivamente, após o diagnóstico. A pressão de átrio direito elevada foi o melhor fator preditivo de mortalidade, não havendo diferença significativa entre a sobrevida dos casos de HP isolada e associada à fibrose pulmonar. Neste estudo, os pacientes eram inicialmente 
submetidos a uma avaliação preliminar com o ECO. Aqueles doentes com PSAP > $35 \mathrm{mmHg}$ ao ECO associada à DLCO $<50 \%$ ou com queda do valor de DLCO de $20 \%$ em relação ao último ano, sem fibrose pulmonar ou com dispnéia sem causa aparente, eram encaminhados para realização de CAT. Os pacientes com CAT evidenciando pressão média de artéria pulmonar $>25 \mathrm{mmHg}$ em repouso ou $>30 \mathrm{mmHg}$ após esforço eram diagnosticados como HP. Cento e sessenta e quatro pacientes apresentaram alterações sugestivas de HP à avaliação com o ECO; destes, 12 não realizaram CAT por idade avançada ou presença de múltiplas co-morbidades e 5 se recusaram a fazê-lo. Dos 147 pacientes que fizeram o CAT, 36 não preencheram os critérios de diagnóstico de HP pelo CAT, enquanto que 22 tinham HP por outros motivos (tromboembolismo pulmonar e HP pós-capilar) $)^{(25)}$.

A história natural da HP na ES ainda não é bem conhecida; acredita-se que, enquanto alguns pacientes apresentam uma evolução ruim, outros apresentam uma doença vascular pulmonar de evolução mais indolente, com elevação dos níveis de pressão pulmonar de forma mais lenta. Embora pareçam existir algumas semelhanças entre a HP idiopática e aquela associada à ES, estas não se aplicam a todos os casos. O melhor conhecimento da história natural da HP relacionada à ES depende não só da padronização de uma rotina de avaliação para o diagnóstico precoce desta complicação, mas também de uma monitorização da sua evolução e resposta terapêutica, baseada em medidas seriadas das pressões em artéria pulmonar paralelas à avaliação do grau de comprometimento funcional ${ }^{(4)}$. Sendo assim, fazse necessário uma reavaliação dos critérios ecocardiográficos utilizados para diagnóstico da HP na ES, com o objetivo de evitar uma superestimativa destes casos e tornar possível a comparação de suas características em diferentes populações e com a HP relacionada às outras doenças do tecido conjuntivo. Vale ressaltar que o ECO é examinador-dependente, ou seja, a sua acurácia está intimamente ligada à experiência do examinador, enquanto o cateterismo cardíaco ainda é o padrão ouro para o diagnóstico da HP na ES, devendo sempre que possível ser realizado para confirmação do diagnóstico da HP na avaliação terapêutica. A combinação de variáveis clínicas, provas de função pulmonar e o ECO, conforme proposto em alguns trabalhos ${ }^{(15,25)}$, pode resultar num critério melhor para o diagnóstico inicial da HP na ES, superando as deficiências do ECO como parâmetro isolado. Isto seria de grande auxílio nos locais onde não existe acesso fácil ao CAT para confirmação do diagnóstico de HP.

O sexto encontro do OMERACT, ao analisar os instrumentos de medida utilizados até o momento nos ensaios clínicos na ES, definiu pontos importantes que complementam estas observações. Sobre a difusão de CO, estabeleceu-se que esta pode ser influenciada tanto pelo acometimento intersticial quanto vascular pulmonar, de forma que ainda não está clara a sua utilidade na diferenciação entre estas duas formas de acometimento pulmonar na ES. O CAT do ventrículo direito, considerado como padrão-ouro para a realização das medidas de pressão em artéria pulmonar, tem seus benefícios bem documentados tanto para o diagnóstico quanto para a avaliação da resposta terapêutica na HP, primária ou associada à ES. No entanto, há uma necessidade de padronização desta técnica entre os diferentes serviços que a utilizam para a avaliação da HP relacionada à ES, para permitir futuras comparações entre coortes. O ECO oferece algumas vantagens em relação ao CAT de ventrículo direito, sendo não invasivo, mais acessível e menos oneroso, porém apresenta limitações na interpretação dos resultados e a medida de PSAP pode não ser possível em $20 \%$ a $30 \%$ dos pacientes. A sua acurácia em relação ao CAT de ventrículo direito foi testada, mas ainda não foram realizados novos testes para a validação da sua sensibilidade na $\mathrm{ES}^{(28)}$.

Em conclusão, a prevalência de HP associada à ES encontrada neste estudo foi semelhante àquelas observadas em outros trabalhos, apesar das limitações nesta comparação. A investigação da HP através do ECO é uma prática acessível, de grande auxílio para o diagnóstico precoce do acometimento vascular pulmonar na ES. No entanto, é necessário que seja revisto o ponto de corte da PSAP medida ao ECO para diagnóstico da HP relacionada à ES, bem como a possibilidade de associação deste parâmetro a outras variáveis ecocardiográficas, clínicas e de função pulmonar, a fim de se padronizar critérios para o diagnóstico e seguimento da HP na ES. 


\section{REFERÊNCIAS}

1. Marques Neto JF, Sampaio-Barros PD: Esclerose Sistêmica. Em Moreira C, Carvalho MAP (Editores): Reumatologia Diagnóstico e Tratamento. $2^{\mathrm{a}}$ edição, MEDSI, Belo Horizonte: 465-88, 2001.

2. Wigley FM: Clinical features of systemic sclerosis. Em Klippel JH, Dieppe PA (Editores): Rheumatology. $2^{\text {a }}$ edição, Mosby, London: 7:9.1-14, 1998

3. Denton CP, Black CM: Pulmonary vascular involvement in systemic sclerosis. Em Clements PJ, Furst DE (Editores): Systemic sclerosis. $2^{a}$ edição, Lippincott Williams \& Wilkins, Filadélfia: 184-94, 2004.

4. Denton CP, Black CM: Pulmonary hypertension in systemic sclerosis. Rheum Dis Clin North Am 29: 371-390, 2003.

5. Denton CP, Cailes JB, Phillipis GD, Wells AU, Black CM, Du Bois RM: Comparison of Doppler echocardiography and right heart catheterization in systemic sclerosis. Br J Rheumatol 36: 239-43, 1997.

6. Subcommittee for Scleroderma Criteria of the American Rheumatism Association Diagnostic and Therapeutic Criteria Committee: Preliminary criteria for the classification of systemic sclerosis (scleroderma). Arthritis Rheum 23: 581-90, 1980.

7. LeRoy EC, Black C, Fleischmajer R, et al: Scleroderma (systemic sclerosis): classification, subsets and pathogenesis. J Rheumatol 15: 202-5, 1988

8. Otto CM: Textbook of clinical echocardiography. $2^{\text {a }}$ edição, W. B. Saunders, Filadélfia, 2000.

9. I Consenso Brasileiro sobre Espirometria 1996. J Bras Pneumol 22: 105-64, 1996

10. Steen V: Predictors of end stage lung disease in systemic sclerosis. Ann Rheum Dis 62: 97-9, 2003

11. Magliano M, Isenberg DA, Hillson J: Pulmonary hypertension in autoimmune rheumatic diseases. Where are we now? Arthritis Rheum 46: 1997-2009, 2002.

12. Vancheeswaran R, Magoulas T, Efrat G et al: Circulating endothelin1 levels in systemic sclerosis subsets - a marker of fibrosis or vascular dysfunction? J Rheumatol 21: 1838-44, 1994.

13. Mitri GM, Lucas M, Fertig N, Steen VD, Medsger TA Jr: A comparison between anti-Th/To and anticentromere antibodypositive systemic sclerosis patients with limited cutaneous involvement. Arthritis Rheum 48: 203-9, 2003.

14. Sacks DG, Okano Y, Steen VD, Curtiss E, Shapiro LS, Medsger TA Jr: Isolated pulmonary hypertension in systemic sclerosis with diffuse cutaneous involvement: association with serum anti-U3RNP antibody. J Rheumatol 23: 639-42, 1996.
15. Steen V, Mesdger TA Jr: Predictors of isolated pulmonary hypertension in patients with systemic sclerosis and limited cutaneous involvement. Arthritis Rheum 48: 516-22, 2003.

16. Yamane K, Ihn H, Asano Y et al: Clinical and laboratory features of scleroderma patients with pulmonary hypertension. Rheumatology 39: 1269-71, 2000

17. Steen V, Conte C, Owens G, Mesdger TA Jr: Severe restrictive lung disease in systemic sclerosis. Arthritis Rheum 37: 1283-9, 1994.

18. Morgan C, Knight C, Lunt M, Black CM, Silman AJ: Predictors of end stage lung disease in a cohort of patients with scleroderma. Ann Rheum Dis 62: 146-50, 2003.

19. Sackner MA, Heinz ER, Steinberg AJ: The heart in scleroderma. Am J Cardiol 17: 542-59, 1966.

20. Stupi AM, Steen V, Owens GR, Barnes EL, Rodnan GP, Mesdger TA Jr: Pulmonary hypertension in the CREST Syndrome variant of systemic sclerosis. Arthritis Rheum 29: 515-24, 1986.

21. Sampaio-Barros PD, Leme Jr. CA, Marques Neto JF: Valor do ecocardiograma com "doppler" na esclerose sistêmica. Rev Bras Reumatol 35: 327-31, 1995

22. Koh ET, Lee P, Gladman DD, Abu-Shakra M: Pulmonary hypertension in systemic sclerosis: an analysis of 17 patients. $\mathrm{Br}$ Rheumatol 35: 989-93, 1996.

23. Battle RW, Davitt MA, Cooper SM et al: Prevalence of pulmonary hypertension in limited and diffuse scleroderma. Chest 110: 1515-9, 1996.

24. Murata I, Takenaka K, Yoshinoya S et al: Clinical evaluation of pulmonary hypertension in systemic sclerosis and related disorders. a Doppler echocardiographic study of 135 Japanese patients. Chest 111: 36-43, 1997.

25. MacGregor AJ, Canavan R, Knight C et al: Pulmonary hypertension in systemic sclerosis: risk factors for progression and consequences for survival. Rheumatology 40: 453-9, 2001

26. Mukerjee D, St George D, Coleiro B et al: Prevalence and outcome in systemic sclerosis associated pulmonary arterial hypertension: application of a registry approach. Ann Rheum Dis 62: 1088-93, 2003.

27. Rich S: World Symposium on Primary Pulmonary Hypertension. 1998, Evian, France. (www.who.int/ncd/cvd/pph.html).

28. Merkel PA, Clements PJ, Reveille JD, Suarez-Almazor ME, Valentini G, Furst DE: Current status of outcome measure development for clinical trials in systemic sclerosis. Report from OMERACT 6. J Rheumatol 30: 1630-47, 2003. 